\title{
PRODUCT FORMULAS AND CONVOLUTIONS FOR THE RADIAL OBLATE SPHEROIDAL WAVE FUNCTIONS*
}

\author{
WILLIAM C. CONNETT ${ }^{\dagger}$, CLEMENS MARKETT ${ }^{\ddagger}$, AND ALAN L. SCHWARTZ $\$$
}

To Richard Askey on his 65th birthday

\begin{abstract}
A product formula is found for the oblate radial spheroidal wave functions. This product formula is then used to define a convolution of measures, and the resulting measure algebras are described. Some of these are new hypergroups. It is shown that a number of historically important non-compact hypergroups can be thought of as special cases of the measure algebras that arise with oblate spheroidal wave functions as characters. This completes the study of spheroidal wave functions begun in [CMS93].
\end{abstract}

1. Introduction. We begin with a brief introduction to the spheroidal wave functions; for background beyond that reviewed here, for a discussion of hypergroups, and for more information on the relation between product formulas and measure algebras the reader should see [CMS93] and the references cited there.

The spheroidal wave functions arise in a natural way when the three-dimensional wave equation $\left(\Delta+k^{2}\right) W=0$ is solved by the separation of variables $W(x, \theta, \phi)=$ $U(x) V(\theta) e^{ \pm i m \phi}$. If confocal families of prolate spheroids and hyperboloids of two sheets are used, this leads to the ordinary differential equations for $U$ and $V$ :

$$
\begin{array}{ll}
U^{\prime \prime}+(\operatorname{coth} x) U^{\prime}-\left[h-(k c \sinh x)^{2}+(m \operatorname{csch} x)^{2}\right] U=0 & (0<x<\infty) \\
V^{\prime \prime}+(\cot \theta) V^{\prime}+\left[h+(k c \sin \theta)^{2}-(m \csc \theta)^{2}\right] V=0 & (0<\theta<\pi) .
\end{array}
$$

When confocal families of a oblate spheroids and hyperboloids of a single sheet are used, this leads the the ordinary differential equations

$$
\begin{array}{ll}
U^{\prime \prime}+(\tanh x) U^{\prime}-\left[h-(k c \cosh x)^{2}-(m \operatorname{sech} x)^{2}\right] U=0 & (0<x<\infty) \\
V^{\prime \prime}+(\cot \theta) V^{\prime}+\left[h-(k c \sin \theta)^{2}-(m \csc \theta)^{2}\right] V=0 & (0<\theta<\pi) .
\end{array}
$$

By a transformation of the dependent variable $V(\theta)=(\sin \theta)^{m} S(\theta)$ the equations (2) and (4) can be written in normalized form

$$
S^{\prime \prime}(\theta)+\frac{2 \alpha+1}{\tan \theta} S^{\prime}(\theta)+\left[\lambda-\gamma \sin ^{2} \theta\right] S(\theta)=0 \quad(0<\theta<\pi)
$$

where $\alpha=m$ and $\gamma=\mp(k c)^{2}$, with $\gamma<0$ for prolate, $\gamma>0$ for oblate. With the boundary condition $S^{\prime}(0)=S^{\prime}(\pi)=0$ and $S(0)=1$ this becomes a Sturm-Liouville problem with a discrete spectrum of eigenvalues. The corresponding eigenfunctions are called angular spheroidal wave functions $\left\{S_{n}^{\alpha, \gamma}(\theta)\right\}_{n \in \mathbb{N}_{0}}, \mathbb{N}_{0}=\{0,1,2, \ldots\}$ for all $\alpha \geq-1 / 2, \gamma \in \mathbb{R}$.

\footnotetext{
${ }^{*}$ Received April 17, 1998, revised Feb 26, 1999.

† Department of Mathematics and Computer Science, University of Missouri-St. Louis, St. Louis, MO 63121, USA (connett@arch.umsl.edu). The research of this author was supported by the National Science Foundation, Grant No. DMS-9706965.

$\ddagger$ Lehrstuhl A für Mathematik, Templergraben 55, D-52062 Aachen, Germany (cmarkett@AMGruppe.de).

$\S$ Department of Mathematics and Computer Science, University of Missouri-St. Louis, St. Louis, MO 63121, U. S. A. (schwartz@arch.umsl.edu). The research of this author was supported by the National Science Foundation, Grant No. DMS-9706965.
} 
If the dependent variable in (1) is transformed using $U(x)=(\sinh x)^{\alpha} \mathcal{S}(x)$, then the equation becomes

(6) $\mathcal{S}^{\prime \prime}(x)+\frac{2 \alpha+1}{\tanh x} \mathcal{S}^{\prime}(x)+\left[\lambda^{2}+(\alpha+1 / 2)^{2}-\gamma \sinh ^{2} x\right] \mathcal{S}(x)=0 \quad(0<x<\infty)$.

The solutions that satisfy the initial conditions $\mathcal{S}(0)=1, \mathcal{S}^{\prime}(0)=0$ are denoted by $\mathcal{S}_{\lambda}^{\alpha, \gamma}(\theta)$, and are called the radial prolate spheroidal wave functions.

If the dependent variable in (3) is transformed using $U(x)=(\cosh x)^{\alpha} \mathcal{T}(x)$, then the equation becomes

(7) $\mathcal{T}^{\prime \prime \prime}(x)+\frac{2 \alpha+1}{\operatorname{coth} x} \mathcal{T}^{\prime}(x)+\left[\lambda^{2}+(\alpha+1 / 2)^{2}+\gamma \cosh ^{2} x\right] \mathcal{T}(x)=0 \quad(0<x<\infty)$.

Note that although (5) and (6) are equations which are regular singular at 0, 0 is a regular point of (7), so more general initial conditions are possible. Define even boundary conditions by:

(8) $\mathcal{T}_{e, \lambda}^{\alpha, \gamma}$ is the solution of $(7)$ which satisfies $\mathcal{T}_{e, \lambda}^{\alpha, \gamma}(0)=1$ and $\left(\mathcal{T}_{e, \lambda}^{\alpha, \gamma}\right)^{\prime}(0)=0$,

and cdd boundary conditions by

(9) $\mathcal{T}_{o, \lambda}^{\alpha, \gamma}$ is the solution of (7) which satisfies $\mathcal{T}_{o, \lambda}^{\alpha, \gamma}(0)=0$ and $\left(\mathcal{T}_{o, \lambda}^{\alpha, \gamma}\right)^{\prime}(0)=1$.

We refer to $\mathcal{T}_{e, \lambda}^{\alpha, \gamma}$ and $\mathcal{T}_{o, \lambda}^{\alpha, \gamma}$ as the even and odd radial oblate spheroidal wave functions. The general solution of (7) is

$$
\mathcal{T}_{\lambda}^{\alpha, \gamma}(x)=a \mathcal{T}_{e, \lambda}^{\alpha, \gamma}(x)+b \mathcal{T}_{o, \lambda}^{\alpha, \gamma}(x)
$$

Further, it should be noted that in the case $\gamma=0$ and $\alpha \neq-1 / 2,(6)$ and (7) are different, i.e.

$$
\begin{aligned}
& \mathcal{S}^{\prime \prime}(x)=\frac{2 \alpha+1}{\tanh x} \mathcal{S}^{\prime}(x)+\left[\lambda^{2}+(\alpha+1 / 2)^{2}\right] \mathcal{S}(x)=0 \\
& \mathcal{T}^{\prime \prime}(x)=\frac{2 \alpha+1}{\operatorname{coth} x} \mathcal{T}^{\prime}(x)+\left[\lambda^{2}+(\alpha+1 / 2)^{2}\right] \mathcal{T}(x)=0 .
\end{aligned}
$$

The solution of (10) is the Jacobi function

$$
\mathcal{S}_{\lambda}^{\alpha, 0}(x)=\phi_{\lambda}^{(\alpha,-1 / 2)}(x)=\phi_{2 \lambda}^{(\alpha, \alpha)}(x / 2) .
$$

Solutions of (11) are also given in terms of Jacobi functions:

$$
\mathcal{T}_{e, \lambda}^{\alpha, 0}(x)=\phi_{\lambda}^{(-1 / 2, \alpha)}(x) \quad \text { and } \quad \mathcal{T}_{o, \lambda}^{\alpha, 0}(x)=\sinh x \phi_{\lambda}^{(1 / 2, \alpha)}(x) .
$$

(see [Koo84, §2.1]). It is shown in [Fle72] that the real bounded solutions of (11) for all $\alpha \geq-1 / 2$ are $\mathcal{T}_{\lambda}^{\alpha, 0}(\theta)$ for

$$
\lambda \in \Lambda_{\alpha}=\{\lambda: \lambda \geq 0 \text { or } \lambda=\mu i \text { with } 0 \leq|\mu| \leq \alpha+1 / 2\}
$$

In an earlier paper with a similar title [CMS93], an explicit product formula was given for the oblate and prolate angular functions, and for the radial prolate functions. 
Both the angular and radial prolate product formulas are given with positive measures, which allows the definition of several new families of hypergroups.

The product formulas for the oblate angular functions have positive measures for a certain range of $(\alpha, \gamma)$, and a similar result with a different range applies to the oblate radial product formula obtained in this article. The product formula is given for all $(\alpha, \gamma)$ with $\alpha \geq-1 / 2$ and $\gamma \geq 0$. These measures are positive for $-1 / 2 \leq \alpha \leq 1 / 2$, $\gamma \geq 0$ and a new family of hypergroups is obtained.

Taken together, the product formulas given in Theorem 2.1 for the oblate case and in [CMS93] for the prolate case allow for a more complete description of the relationship between the product formula given in [FK73] for the Jacobi functions and the spheroidal wave functions. The results obtained for the prolate case and the odd oblate case yield the same results as in [FK73] by a different method; the results for the even oblate yields an extension of the results in [FK73], and are described in the corollary following Theorem 2.1.

2. Product formulas. The proof is based on an explicit formula for the Riemann function given in a paper of Henrici [Hen57] on the work of Vekua, and an application of Green's Theorem. The argument is in many ways easier than the argument in [CMS93], because of the lack of singularity along the critical boundary, so what follows can be viewed as both a primer to and a completion of the argument given there.

THEOREM 2.1 (Product formulas for oblate radial functions). Let $\alpha \geq-1 / 2$, $\gamma \geq 0, \lambda \in \Lambda_{\alpha}$, and $0 \leq x, y<\infty$, then

$$
\begin{aligned}
& \mathcal{T}_{e, \lambda}^{\alpha, \gamma}(x) \mathcal{T}_{e, \lambda}^{\alpha, \gamma}(y) \\
&=\frac{1}{2}\left[\frac{\cosh (x-y)}{\cosh x \cosh y}\right]^{\alpha+1 / 2} \mathcal{T}_{e, \lambda}^{\alpha, \gamma}(|x-y|)+\frac{1}{2}\left[\frac{\cosh (x+y)}{\cosh x \cosh y}\right]^{\alpha+1 / 2} \mathcal{T}_{e, \lambda}^{\alpha, \gamma}(x+y) \\
&+\int_{|x-y|}^{x+y} \mathcal{T}_{e, \lambda}^{\alpha, \gamma}(\xi) \mathcal{K}_{o b l}^{\alpha, \gamma}(\xi ; x, y)(\cosh \xi)^{2 \alpha+1} d \xi
\end{aligned}
$$

and

$$
\mathcal{T}_{o, \lambda}^{\alpha, \gamma}(x) \mathcal{T}_{o, \lambda}^{\alpha, \gamma}(y)=\int_{|x-y|}^{x+y} \mathcal{T}_{o, \lambda}^{\alpha, \gamma}(\xi) \mathcal{M}_{o b l}^{\alpha, \gamma}(\xi ; x, y)(\cosh \xi)^{2 \alpha+1} d \xi
$$

where $\mathcal{K}_{o b l}^{\alpha, \gamma}(\xi ; x, y)$ and $\mathcal{M}_{o b l}^{\alpha, \gamma}(\xi ; x, y)$ are supported on $|x-y| \leq \xi \leq x+y$ with the values

$$
\begin{aligned}
& \mathcal{K}_{o b l}^{\alpha, \gamma}(\xi ; x, y) \\
& =\frac{\sinh \xi \sinh x \sinh y}{4(\cosh \xi \cosh x \cosh y)^{\alpha+1 / 2}} \sum_{\substack{k, n=0 \\
k+n \neq 0}}^{\infty} \frac{\left(\frac{1}{2}+\alpha\right)_{n}\left(\frac{1}{2}-\alpha\right)_{n}}{k ! n !(k+n-1) !} \gamma^{k} \Omega_{0}^{-n}\left(\frac{\chi_{0}}{4}\right)^{k+n-1}
\end{aligned}
$$

(15) $\mathcal{M}_{o b l}^{\alpha, \gamma}(\xi ; x, y)$

$$
=\frac{1}{2}(\cosh \xi \cosh x \cosh y)^{-\alpha-1 / 2} \sum_{k, n=0}^{\infty} \frac{\left(\frac{1}{2}+\alpha\right)_{n}\left(\frac{1}{2}-\alpha\right)_{n}}{k ! n !(k+n) !} \gamma^{k} \Omega_{0}^{-n}\left(\frac{\chi_{0}}{4}\right)^{k+n},
$$


where

$$
\begin{aligned}
& \Omega_{0}=\Omega_{0}(\xi ; x, y)=\cosh \xi \cosh x \cosh y \\
& \chi_{0}=\chi_{0}(\xi ; x, y)=[\cosh (x+y)-\cosh \xi][\cosh \xi-\cosh (x-y)]
\end{aligned}
$$

Corollary 2.2 (Product formulas for Jacobi functions). For $\gamma=0$

(i) $\mathcal{T}_{e, \lambda}^{\alpha, 0}(x)=\phi_{\lambda}^{(-1 / 2, \alpha)}(x)$, and (14) is an extension of the product formula in [FK'7] to the range $\alpha>-1 / 2$ with

$$
\begin{aligned}
\mathcal{K}_{o b l}^{\alpha, 0}(\xi ; x, y)= & \frac{\sinh \xi \sinh x \sinh y}{4(\cosh \xi \cosh x \cosh y)^{\alpha+1 / 2}} \sum_{n=1}^{\infty} \frac{\left(\frac{1}{2}+\alpha\right)_{n}\left(\frac{1}{2}-\alpha\right)_{n}}{n !(n-1) !} \Omega_{0}^{-n}\left(\frac{\chi_{0}}{4}\right)^{n-1} \\
=\left(1 / 4-\alpha^{2}\right) & \frac{\sinh \xi \sinh x \sinh y}{4(\cosh \xi \cosh x \cosh y)^{\alpha+3 / 2}} \\
& \times{ }_{2} F_{1}\left(3 / 2+\alpha, 3 / 2-\alpha ; 2 ; \chi_{0} /\left(4 \Omega_{0}\right)\right)
\end{aligned}
$$

positive for $|\alpha| \leq 1 / 2$.

(ii) $\mathcal{T}_{o, \lambda}^{\alpha, 0}(x)=\sinh x \phi_{\lambda}^{(1 / 2, \alpha)}(x)$, and (13) yields the product formula in [FK73] which is positive for $|\alpha| \leq 1 / 2$ with the kernel

$$
\begin{aligned}
\mathcal{M}_{o b l}^{\alpha, 0}(\xi ; x, y) & =\frac{1}{2(\cosh \xi \cosh x \cosh y)^{\alpha+1 / 2}} \sum_{n=0}^{\infty} \frac{\left(\frac{1}{2}+\alpha\right)_{n}\left(\frac{1}{2}-\alpha\right)_{n}}{(n !)^{2}}\left(\frac{\chi_{0}}{4 \Omega_{0}}\right)^{n} \\
& =\frac{1}{2(\cosh \xi \cosh x \cosh y)^{\alpha+1 / 2}}{ }_{2} F_{1}\left(1 / 2+\alpha, 1 / 2-\alpha ; 1 ; \chi_{0} /\left(4 \Omega_{0}\right)\right) .
\end{aligned}
$$

Remark. It was shown in [CMS93, Theorem 2] that in the prolate case $\mathcal{S}_{\lambda}^{\alpha, 0}=$ $\phi_{\lambda}^{(\alpha,-1 / 2)}$, and the product formula has a positive kernel for all $\alpha \geq-1 / 2$.

Proof. [Proof of Theorem] The cases $x=0$ and $y=0$ are trivial, so by symmetry it suffices to consider $x>y>0$. Let

$$
L_{\xi}^{\alpha, \gamma}=\frac{\partial^{2}}{\partial \xi^{2}}+(2 \alpha+1)(\tanh \xi) \frac{\partial}{\partial \xi}+\gamma \cosh ^{2} \xi
$$

then

$$
L_{\xi}^{\alpha, \gamma} \mathcal{T}_{e, \lambda}^{\alpha, \gamma}(\xi)=-\left[\lambda^{2}+(\alpha+1 / 2)^{2}\right] \mathcal{T}_{e, \lambda}^{\alpha, \gamma}(\xi)
$$

Use a similar definition for $L_{\eta}^{\alpha, \gamma}$; then $u(\xi, \eta)=\mathcal{T}_{e, \lambda}^{\alpha, \gamma}(\xi) \mathcal{T}_{e, \lambda}^{\alpha, \gamma}(\eta)$ for $0 \leq \xi, \eta<\infty$ is a solution to the hyperbolic equation

$$
\left(L_{\xi}^{\alpha, \gamma}-L_{\eta}^{\alpha, \gamma}\right) u(\xi, \eta)=0 .
$$

The even initial conditions (8) imply the boundary conditions

$$
u(\xi, 0)=\mathcal{T}_{e, \lambda}^{\alpha, \gamma}(\xi) \text { and } u_{\eta}(\xi, 0)=0
$$

Define the triangle

$$
\Delta_{x, y}=\{(\xi, \eta): x-y \leq \xi-\eta \leq \xi+\eta \leq x+y\} .
$$


$\Delta_{x, y}$ has vertices $P=(x, y), Q=(|x-y|, 0)$ and $R=(x+y, 0)$. The Riemann function is the unique solution $v(\xi, \eta)$ to the adjoint equation and characteristic boundary conditions on the triangle $\Delta_{x, y}$ :

$$
\begin{aligned}
\left(L_{\xi}^{\alpha, \gamma}-\right. & \left.L_{\eta}^{\alpha, \gamma}\right)^{*} v(\xi, \eta)=v_{\xi \xi}-v_{\eta \eta}-[(2 \alpha+1)(\tanh \xi) v]_{\xi} \\
& +[(2 \alpha+1)(\tanh \eta) v]_{\eta}+\gamma\left[\cosh ^{2} \xi-\cosh ^{2} \eta\right] v=0 \quad\left((\xi, \eta) \in \Delta_{x, y}\right)
\end{aligned}
$$

with boundary conditions

$$
v_{\xi} \pm v_{\eta}-(\alpha+1 / 2)[\tanh \xi \pm \tanh \eta] v=0 \quad \text { on } \xi \mp \eta=x \mp y,
$$

and with normalization

$$
v(x, y)=1
$$

An actual formula for the Riemann function is given in Henrici [Hen57] in terms of the "characteristic" co-ordinates

$$
X=\cosh (\xi+\eta), \quad Y=\cosh (\xi-\eta), \quad X_{0}=\cosh (x+y), \quad Y_{0}=\cosh (x-y) .
$$

We also need

$$
\begin{aligned}
& \Omega=\Omega(\xi, \eta ; x, y)=\cosh \xi \cosh \eta \cosh x \cosh y \\
& \chi=\chi(\xi, \eta ; x, y)=[\cosh (x+y)-\cosh (\xi+\eta)][\cosh (\xi-\eta)-\cosh (x-y)] .
\end{aligned}
$$

Note that when $(\xi, \eta) \in \Delta_{x, y}, 0<Y_{0} \leq Y \leq X \leq X_{0}$. In terms of these co-ordinates

$$
v(\xi, \eta)=R_{o b l}^{\alpha, \gamma}(\xi, \eta ; x, y)=\left(\frac{X+Y}{X_{0}+Y_{0}}\right)^{\alpha+1 / 2} V\left(X, Y ; X_{0}, Y_{0}\right)
$$

where $V$ is given explicitly as the solution of a characteristic boundary value problem:

$$
V_{X Y}+\left[\frac{\frac{1}{4}-\alpha^{2}}{(X+Y)^{2}}+\frac{\gamma}{4}\right] V=0
$$

with the following boundary conditions obtained from (19) and (20)

$$
V\left(X, Y ; X_{0}, Y_{0}\right)=1 \text { if } X=X_{0} \text { or } Y=Y_{0} .
$$

The solution of (22) and (23) is given by

$$
\begin{aligned}
V & =\Xi_{2}\left(1 / 2+\alpha, 1 / 2-\alpha, 1, W_{3},-\gamma W_{1} / 4\right) \\
& =\sum_{k, n=0}^{\infty} \frac{\left(\frac{1}{2}+\alpha\right)_{n}\left(\frac{1}{2}-\alpha\right)_{n}}{k ! n !(k+n) !} W_{3}^{n}\left(-\frac{\gamma}{4} W_{1}\right)^{k}
\end{aligned}
$$

where

$$
\begin{aligned}
W_{1} & =\left(X-X_{0}\right)\left(Y-Y_{0}\right) \\
& =-[\cosh (x+y)-\cosh (\xi+\eta)][\cosh (\xi-\eta)-\cosh (x-y)]=-\chi \\
W_{3} & =\frac{\left(X_{0}-X\right)\left(Y-Y_{0}\right)}{(X+Y)\left(X_{0}+Y_{0}\right)}=\frac{\chi}{4 \Omega} .
\end{aligned}
$$


See [EMOT53, 5.7.1(26)] for $\Xi_{2}$. We now show that the double sum (24) converges uniformly for $(\xi, \eta) \in \Delta_{x, y}$. First we note that

$$
0 \leq \chi=\left(X_{0}-X\right)\left(Y-Y_{0}\right)<\left(X_{0}-Y_{0}\right)^{2} \text {. }
$$

So on the triangle $\nabla x_{0}, Y_{0}=\left\{(X, Y): 0<Y_{0} \leq Y \leq X \leq X_{0}\right\}, W_{3} \geq 0$. Also $2 X Y+2 X_{0} Y_{0}+(X-Y)\left(X_{0}-Y_{0}\right) \geq 0$ from which it follows that $2\left(X_{0}-X\right)\left(Y-Y_{0}\right) \leq$ $(X+Y)\left(X_{0}+Y_{0}\right)$ thus

$$
0 \leq W_{3} \leq \frac{1}{2}
$$

Now

$$
\begin{aligned}
\mid \sum_{k, n=0}^{\infty} & \frac{\left(\frac{1}{2}+\alpha\right)_{n}\left(\frac{1}{2}-\alpha\right)_{n}}{k ! n !(k+n) !}(\gamma \chi / 4)^{k} W_{3}^{n} \mid \\
& \leq \sum_{n=0}^{\infty} \frac{\left(\frac{1}{2}+\alpha\right)_{n}\left(\left|\frac{1}{2}-\alpha\right|\right)_{n}}{2^{n} n !} \sum_{k=0}^{\infty} \frac{1}{k !(k+n) !}(\gamma \chi / 4)^{k} \\
& \leq \sum_{n=0}^{\infty} \frac{\left(\frac{1}{2}+\alpha\right)_{n}\left(\left|\frac{1}{2}-\alpha\right|\right)_{n}}{2^{n}(n !)^{2}} \sum_{k=0}^{\infty} \frac{1}{k !}(\gamma \chi / 4)^{k} \\
& \leq\left[\sum_{n=0}^{\infty} \frac{\left(\frac{1}{2}+\alpha\right)_{n}\left(\left|\frac{1}{2}-\alpha\right|\right)_{n}}{2^{n}(n !)^{2}}\right] e^{\frac{\gamma}{4}\left(X_{0}-Y_{0}\right)^{2}} .
\end{aligned}
$$

The hypergeometric series converges by the ratio test, so we obtain the bound

$$
V\left(X, Y ; X_{0}, Y_{0}\right) \leq A_{\alpha} e^{\frac{\gamma}{4}\left(X_{0}-Y_{0}\right)^{2}} \leq A_{\alpha} \exp \left[\frac{\gamma}{16} e^{2 x+2 y}\right]
$$

which is independent of $\xi$ and $\eta$, and where $A_{\alpha}={ }_{2} F_{1}(1 / 2+\alpha,|1 / 2-\alpha| ; 1 ; 1 / 2)$.

Changing back to the original variables and using the notation in (21), we obtain

$$
R_{o b l}^{\alpha, \gamma}(\xi, \eta ; x, y)=\left(\frac{\cosh \xi \cosh \eta}{\cosh x \cosh y}\right)^{\alpha+1 / 2} \sum_{k, n=0}^{\infty} \frac{\left(\frac{1}{2}+\alpha\right)_{n}\left(\frac{1}{2}-\alpha\right)_{n}}{k ! n !(k+n) !} \gamma^{k} \Omega^{-n}\left(\frac{\chi}{4}\right)^{k+n}
$$

Some specific properties of $R_{o b l}^{\alpha, \gamma}(\xi, \eta ; x, y)$ will be needed. When $\eta=0$,

$$
R_{o b l}^{\alpha, \gamma}(\xi, 0 ; x, y)=\left(\frac{\cosh \xi}{\cosh x \cosh y}\right)^{\alpha+1 / 2} \sum_{k, n=0}^{\infty} \frac{\left(\frac{1}{2}+\alpha\right)_{n}\left(\frac{1}{2}-\alpha\right)_{n}}{k ! n !(k+n) !} \gamma^{k} \Omega_{0}^{-n}\left(\frac{\chi_{0}}{4}\right)^{k+n}
$$

Notice that

$$
R_{o b l}^{\alpha, \gamma}(x \pm y, 0 ; x, y)=\left(\frac{\cosh (x \pm y)}{\cosh x \cosh y}\right)^{\alpha+1 / 2} .
$$

The final fact about the Riemann function that is needed is the value of its derivative with respect to $\eta$ along the $\eta=0$ boundary. To this end,

$$
\begin{aligned}
& \left.\frac{\partial \Omega}{\partial \eta}\right|_{\eta=0}=\left.\cosh \xi \sinh \eta \cosh x \cosh y\right|_{\eta=0}=0 \\
& \left.\frac{\partial \chi}{\partial \eta}\right|_{\eta=0}=-2 \sinh \xi \sinh x \sinh y .
\end{aligned}
$$


So that

$$
\begin{aligned}
\left.\frac{\partial}{\partial \eta} R_{o b l}^{\alpha, \gamma}(\xi, \eta ; x, y)\right|_{\eta=0}= & \left.\frac{\cosh \xi}{\cosh x \cosh y}\right)^{\alpha+1 / 2}(-2 \sinh \xi \sinh x \sinh y) \\
& \times \frac{1}{4} \sum_{\substack{k, n=0 \\
k+n \neq 0}}^{\infty} \frac{\left(\frac{1}{2}+\alpha\right)_{n}\left(\frac{1}{2}-\alpha\right)_{n}}{k ! n !(k+n-1) !} \gamma^{k} \Omega_{0}^{-n}\left(\frac{\chi_{0}}{4}\right)^{k+n-1}
\end{aligned}
$$

With this explicit representation of the Riemann function and its derivative, Green's Theorem will now yield the result. With $u$ and $v$ as defined in (16) - (20)

$$
v\left(L_{\xi}^{\alpha, \gamma}-L_{\eta}^{\alpha, \gamma}\right) u-u\left(L_{\xi}^{\alpha, \gamma}-L_{\eta}^{\alpha, \gamma}\right)^{*} v=0
$$

consequently

$$
\begin{aligned}
& 0=\iint_{\Delta_{x, y}}\left[v\left(L_{\xi}^{\alpha, \gamma}-L_{\eta}^{\alpha, \gamma}\right) u-u\left(L_{\xi}^{\alpha, \gamma}-L_{\eta}^{\alpha, \gamma}\right)^{*} v\right] d \xi d \eta \\
&=\iint_{\Delta_{x, y}}\left[\frac{\partial H}{\partial \xi}-\frac{\partial K}{\partial \eta}\right] d \xi d \eta=\oint_{\partial \Delta_{x, y}} K d \xi+H d \eta
\end{aligned}
$$

with

$$
\begin{aligned}
& H=v u_{\xi}-u v_{\xi}+(2 \alpha+1) \tanh \xi v u \\
& K=v u_{\eta}-u v_{\eta}+(2 \alpha+1) \tanh \eta v u
\end{aligned}
$$

and

(31) $K d \xi+H d \eta$

$$
=\left(v u_{\eta}-u v_{\eta}\right) d \xi+\left(v u_{\xi}-u v_{\xi}\right) d \eta+(2 \alpha+1) u v(\tanh \eta d \xi+\tanh \xi d \eta) .
$$

Now

$$
\oint_{\partial \Delta_{x, y}}=\int_{R}^{P}+\int_{P}^{Q}+\int_{Q}^{R} .
$$

On $\overline{R P}$ one has $d \xi=-d \eta$ and by the boundary condition (19)

$$
v_{\xi}-v_{\eta}-(\alpha+1 / 2)[\tanh \xi-\tanh \eta] v=0
$$

similarly on $\overline{P Q}$ one has $d \xi=d \eta$ and

$$
v_{\xi}+v_{\eta}-(\alpha+1 / 2)[\tanh \xi+\tanh \eta] v=0 .
$$

Thus

$$
\int_{R}^{P}(K d \xi+H d \eta)=-\int_{R}^{P}(u v)_{\eta} d \eta+(u v)_{\xi} d \xi=-u(P) v(P)+u(R) v(R)
$$

and

$$
\int_{P}^{Q}(K d \xi+H d \eta)=u(Q) v(Q)-u(P) v(P)
$$


and fir.ally

$$
\int_{Q}^{R}(K d \xi+H d \eta)=\int_{Q}^{R}\left[v u_{\eta}-u v_{\eta}+(2 \alpha+1) \tanh \eta v u\right] d \xi .
$$

Using the boundary conditions (17) and the normalization $v(P)=1,(30)$ becomes

$$
0=-2 u(P)+u(Q) v(Q)+u(R) v(R)-\int_{x-y}^{x+y} u(\xi, 0) v_{\eta}(\xi, 0) d \xi
$$

which is equivalent to (12), with $\mathcal{K}_{o b l}^{\alpha, \gamma}(\xi ; x, y)=-\left.\frac{1}{2} \frac{\partial}{\partial \eta} R_{o b l}^{\alpha, \gamma}(\xi, \eta ; x, y)\right|_{\eta=0}$.

Now we turn to the odd radial oblate functions. The argument for this case uses the Riemann function and Green's Theorem as in the even case, and proceeds along identical lines until (32). Now employ (9) to obtain

$$
u(\xi, 0)=0 \quad \text { and } \quad u_{\eta}(\xi, 0)=\mathcal{T}_{o, \lambda}^{\alpha, \gamma}(\xi)
$$

and use this instead of (17) to simplify (32); it follows that

$$
0=-2 u(P) v(P)+u(Q) v(Q)+u(R) v(R)+\left.\int_{x-y}^{x+y} v u_{\eta}\right|_{\eta=0} d \xi
$$

But $u(Q)=u(R)=0, v(\xi, 0)=R_{o b l}^{\alpha, \gamma}(\xi, 0 ; x, y)$, so (33) yields (13), by setting $\mathcal{M}_{o b l}^{\alpha, \gamma}(\xi ; x, y)=\frac{1}{2} R_{o b l}^{\alpha, \gamma}(\xi, 0 ; x, y)$. tions:

3. Positivity, bounds, and measure algebras. We require several defini-

$C_{B}$ is the space of bounded continuous functions on $[0, \infty)$,

$M$ denotes the space of bounded measures on $[0, \infty)$,

$M_{+}$consists of the non-negative measures in $M$,

$M_{c}$ is the space of compactly supported measures in $M$, and

$M_{1}$ is the set of probability measures in $M$ (positive with unit total variation).

$\delta_{x}$ denotes a unit mass concentrated at $x . M$ is a Banach space with the total variation norm $\|\cdot\|$. The bounded continuous functions on $[0, \infty)$ will be denoted $C_{B}$. If $\mu_{n} \in M$, we say that $\mu_{n}$ converges weakly to $\mu \in M$ if $\lim _{n \rightarrow \infty} \int f d \mu_{n}=\int f d \mu$ for every $f \in C_{B}$.

We begin with some evaluations and observations on positivity and zeros of the radial ciblate spheroidal wave functions.

LEMMA 3.1.

(i) For all $x \in[0, \infty)$

$$
\begin{aligned}
& \mathcal{T}_{e, i(\alpha+1 / 2)}^{\alpha, 0}(x)=1 \\
& \mathcal{T}_{o, i(\alpha+1 / 2)}^{\alpha, 0}(x)=\int_{0}^{x}(\cosh s)^{-2 \alpha-1} d s .
\end{aligned}
$$

(ii) For $|\alpha| \leq 1 / 2$ and $\lambda=0$

$$
\mathcal{T}_{e, 0}^{\alpha, 0}(x)=(\cosh x)^{-\alpha-1 / 2}{ }_{2} F_{1}\left(1 / 4+\alpha / 2,1 / 4-\alpha / 2 ; 1 / 2 ; \tanh ^{2} x\right)>0 .
$$


(iii) Suppose $\lambda=\mu i$ with $\mu^{2} \geq \alpha^{2}-1 / 4$, then

$$
(\cosh x)^{\alpha+1 / 2} \mathcal{T}_{e, \lambda}^{\alpha, 0}(x) \quad \text { and } \quad(\cosh x)^{\alpha+1 / 2} \mathcal{T}_{o, \lambda}^{\alpha, 0}(x)
$$

are positive non-decreasing functions on $(0, \infty)$. If $\mu>0$, then each of $\mathcal{T}_{e, \lambda}^{\alpha, 0}$ and $\mathcal{T}_{o, \lambda}^{\alpha, 0}$ have only finitely many zeros in $(0, \infty)$.

(iv) If $\gamma>0$ or $\lambda>0$, then $\mathcal{T}_{e, \lambda}^{\alpha, \gamma}$ and $\mathcal{T}_{o, \lambda}^{\alpha, \gamma}$ each have infinitely many zeros in $(0, \infty)$.

Proof. (i) (34) and (35) are obtained by solving the differential equation (7). To obtain the other results, we transform the differential equation into Liouville normal form by setting $u(x)=(\cosh x)^{\alpha+1 / 2} \mathcal{T}(x)$ :

$$
u^{\prime \prime}+\left(\lambda^{2}+\frac{\alpha^{2}-1 / 4}{\cosh ^{2} x}+\gamma \cosh ^{2} x\right) u=0
$$

(ii) Since $\mathcal{T}_{e, 0}^{\alpha, 0}=\phi_{0}^{(-1 / 2, \alpha)}$, [Koo84, (2.7)] with $\alpha$ replaced by $-1 / 2$ and $\beta$ replaced by $\alpha$, and $\lambda=0$ yields the result.

(iii) This follows by examining the sign of the coefficient of $u$ in the differential equation.

(iv) This follows from the Sturm comparison theorem.

The product formulas (12) and (13) can be written

$$
\mathcal{T}(x) \mathcal{T}(y)=\int \mathcal{T} d \sigma
$$

where $\mathcal{T}=\mathcal{T}_{\kappa, \lambda}^{\alpha, \gamma}$ and $\sigma=\sigma_{\kappa, x, y}^{\alpha, \gamma}$ with $\alpha \geq-1 / 2, \gamma \geq 0, \kappa \in\{e, o\}, \lambda \in \mathbb{C}$, and $x, y \in[0, \infty)$. The measures are given by their action on $f \in C_{B}$ by

$$
\begin{gathered}
\int f d \sigma_{e, x, y}^{\alpha, \gamma}=\frac{f(|x-y|)}{2}\left[\frac{\cosh (x-y)}{\cosh x \cosh y}\right]^{\alpha+1 / 2}+\frac{f(x+y)}{2}\left[\frac{\cosh (x+y)}{\cosh x \cosh y}\right]^{\alpha+1 / 2} \\
+\int_{|x-y|}^{x+y} f(\xi) \mathcal{K}_{o b l}^{\alpha, \gamma}(\xi ; x, y)(\cosh \xi)^{2 \alpha+1} d \xi
\end{gathered}
$$

and

$$
\int f d \sigma_{o, x, y}^{\alpha, \gamma}=\int_{|x-y|}^{x+y} f(\xi) \mathcal{M}_{o b l}^{\alpha, \gamma}(\xi ; x, y)(\cosh \xi)^{2 \alpha+1} d \xi .
$$

With these definitions we have:

Theorem 3.2. For each $x, y \in[0, \infty)$

(i) If $\gamma \geq 0, \alpha \geq-1 / 2$, and $\kappa \in\{e, o\}$, $\operatorname{supp} \sigma_{\kappa, x, y}^{\alpha, \gamma} \subset[|x-y|, x+y]$; if $M$ is given the weak topology then $(x, y) \mapsto \sigma_{\kappa, x, y}^{\alpha, \gamma}$ is continuous.

(ii) If $\gamma \geq 0, \alpha \geq-1 / 2$, and $\kappa \in\{e, o\}$, then there is an increasing function $B$ on $[0, \infty)$ such that

$$
\left\|\sigma_{\kappa, x, y}^{\alpha, \gamma}\right\| \leq B(x+y) .
$$

(iii) If $\gamma=0, \alpha \geq-1 / 2$ and $\kappa \in\{e, o\}$, then there is a constant $B$ such that

$$
\left\|\sigma_{\kappa, x, y}^{\alpha, 0}\right\| \leq B
$$


(iv) If $\gamma \geq 0,-1 / 2 \leq \alpha \leq 1 / 2$, and $\kappa \in\{e, o\}$ then

$$
\sigma_{\kappa, x, y}^{\alpha, \gamma} \in M_{+}
$$

these measures are strictly positive with the exception that $\sigma_{o, x, 0}^{\alpha, 0}=\sigma_{o, 0, y}^{\alpha, 0}=0$.

(v) If $\gamma=0,-1 / 2 \leq \alpha \leq 1 / 2$, and $\kappa=e$, then

$$
\sigma_{e, x, y}^{\alpha, 0} \in M_{1} \text {. }
$$

(vi) If $\gamma=0,-1 / 2 \leq \alpha \leq 1 / 2, \kappa \in\{e, o\}$, and $\lambda=\mu i$ with $\mu^{2} \geq \alpha^{2}-1 / 4$, then (with the restriction that $x \neq y$ when $\kappa=o$ )

$$
\left\|\sigma_{\kappa, x, y}^{\alpha, 0}\right\| \leq \frac{\mathcal{T}_{\kappa, \lambda}^{\alpha, 0}(x) \mathcal{T}_{\kappa, \lambda}^{\alpha, 0}(y)}{\mathcal{T}_{\kappa, \lambda}^{\alpha, 0}(|x-y|)}\left[\frac{\cosh (x+y)}{\cosh (x-y)}\right]^{\alpha+1 / 2}
$$

(vii) If $\gamma=0,-1 / 2 \leq \alpha \leq 1 / 2$, and $\kappa=o$, then for every $\epsilon>0$, there is $B>0$ such that

$$
\left\|\sigma_{o, x, y}^{\alpha, 0}\right\| \leq(1+\epsilon)\left[\frac{2^{2 \alpha+1}}{2 \alpha+1}\right]^{2} \max \left[\frac{1}{|x-y|}, B\right] .
$$

(viii) If $\gamma=0, \alpha>1 / 2$, and $\kappa=e$, then

$$
\sigma_{e, x, y}^{\alpha, 0} \notin M_{+} .
$$

Procf. (i) The assertion about support follows from (37) and (38). If $M$ is given the weak topology and $f \in C_{B}$, then the mapping $(x, y) \mapsto \int f d \sigma_{x, y}$ is continuous, so the second assertion follows.

(ii) This follows from (14), (15), and (25) (with a small modification for $\kappa=e$ ).

(iii) For $\alpha \geq 1 / 2$, the argument is similar to 2 ; for $-1 / 2 \leq \alpha \leq 1 / 2$ see 5 below.

(iv) The double series that define $\mathcal{K}_{o b l}^{\alpha, \gamma}(\xi ; x, y)$ and $\mathcal{M}_{o b l}^{\alpha, \gamma}(\xi ; x, y)$ are convergent series of non-negative terms because $\chi_{0}$ and $\Omega_{0}$ are non-negative and $(1 / 2+\alpha)_{n}(1 / 2-$ $\alpha)_{n} \geq 0$ when $-1 / 2 \leq \alpha \leq 1 / 2$.

(v) Since $\sigma_{e, x, y}^{\alpha, 0} \in M_{+},(34)$ can be used in (12) to obtain $\left\|\sigma_{e, x, y}^{\alpha, 0}\right\|=1$.

(vi) Recall from Lemma 3.1 that $(\cosh x)^{\alpha+1 / 2} \mathcal{T}_{\kappa, \lambda}^{\alpha, 0}(x)$ is a positive increasing function, so by (36) and (iv)

$$
\begin{aligned}
\mathcal{T}_{\kappa, \lambda}^{\alpha, 0}(x) \mathcal{T}_{\kappa, \lambda}^{\alpha, 0}(y) & =\int_{|x-y|}^{x+y} \frac{\mathcal{T}_{\kappa, \lambda}^{\alpha, 0}(\xi)(\cosh \xi)^{\alpha+1 / 2}}{(\cosh \xi)^{\alpha+1 / 2}} d \sigma_{\kappa, x, y}^{\alpha, 0}(\xi) \\
& \geq \mathcal{T}_{\kappa, \lambda}^{\alpha, 0}(|x-y|)(\cosh |x-y|)^{\alpha+1 / 2} \int_{|x-y|}^{x+y} \frac{1}{(\cosh \xi)^{\alpha+1 / 2}} d \sigma_{\kappa, x, y}^{\alpha, 0}(\xi) \\
& \geq \mathcal{T}_{\kappa, \lambda}^{\alpha, 0}(|x-y|)\left[\frac{\cosh |x-y|}{\cosh (x+y)}\right]^{\alpha+1 / 2}\left\|\sigma_{\kappa, x, y}^{\alpha, 0}\right\| .
\end{aligned}
$$

(vii) Let $\mathcal{T}=\mathcal{T}_{o, i(\alpha+1 / 2)}^{\alpha, 0}=\int_{0}^{x}(\cosh s)^{-2 \alpha-1} d s$ and let $\epsilon>0$; let $c=1+\epsilon . b$ can be chosen so that $(\cosh b)^{2 \alpha+1}=c$, whence

$$
\mathcal{T}(x)> \begin{cases}x / c & \text { if } 0<x \leq b \\ b / c & \text { if } x>b .\end{cases}
$$


We also have

$$
\mathcal{T}(x)<\int_{0}^{\infty}(\cosh s)^{-2 \alpha-1} d s<\frac{2^{2 \alpha+1}}{2 \alpha+1}
$$

Now $\mathcal{T}$ is an increasing function, so when $x \neq y$ the product formula (36) with $\lambda=i(\alpha+1 / 2)$ yields

$$
\left\|\sigma_{x, y}\right\|<\frac{\mathcal{T}(x) \mathcal{T}(y)}{\mathcal{T}(|x-y|)}
$$

and the result follows.

(viii) First note that when $\alpha>1 / 2$

$$
\frac{1}{2}\left[\frac{\cosh (x-y)}{\cosh x \cosh y}\right]^{\alpha+1 / 2}+\frac{1}{2}\left[\frac{\cosh (x+y)}{\cosh x \cosh y}\right]^{\alpha+1 / 2}>1 \quad(x, y \neq 0) .
$$

To see this let $t=\tanh x \tanh y$ and observe that the left-hand side of the inequality can be written

$$
\frac{1}{2}(1-t)^{\alpha+1 / 2}+\frac{1}{2}(1+t)^{\alpha+1 / 2}>1 .
$$

Now recall (34) to see that (12) with $\lambda=i(\alpha+1 / 2)$ and (39) imply

$$
\int_{|x-y|}^{x+y} \mathcal{K}_{o b l}^{\alpha, 0}(\xi ; x, y)(\cosh \xi)^{2 \alpha+1} d \xi<0 .
$$

3.1. Measure algebras. We will generally suppress superscripts and subscripts in this and the next section. The product formula (36) can be used to define an operation $*=*_{\kappa}^{\alpha, \gamma}$, called convolution, on $M$ by its action on $f \in C_{B}$ :

$$
\int f d(\mu * \nu)=\iint\left(\int f d \sigma_{x, y}\right) d \mu(x) d \nu(y) \quad(\mu, \nu \in M) .
$$

* is the unique weakly continuous convolution which satisfies

$$
\delta_{x} * \delta_{y}=\sigma_{x, y} .
$$

It is easy to see from the explicit formulas (14) and (15) that $\sigma_{x, y}=\sigma_{y, x}$ so that $*$ is commutative. Define

$$
\Lambda=\left\{\lambda: \mathcal{T}_{\lambda} \text { is a bounded real-valued function }\right\},
$$

and let

$$
\widehat{\mu}(\lambda)=\int \mathcal{T}_{\lambda} d \mu \quad(\lambda \in \Lambda)
$$

(36) and (40) imply

$$
\widehat{(\mu * \nu)}(\lambda)=\widehat{\mu}(\lambda) \widehat{\nu}(\lambda) \quad(\lambda \in \Lambda) .
$$

When $\gamma=0, \mu \mapsto \widehat{\mu}$ is injective [Koo84, Thm. 2.3], so it follows that $*$ is associative, thus we obtain the following consequences of Theorem 3.2 :

THEOREM 3.3 . 
(i) If $\gamma \geq 0, \alpha \geq-1 / 2$, and $\kappa \in\{e, o\}$ then $*$ is a commutative operation on $M_{c}$. Moreover, there is an increasing function $B$ on $[0, \infty)$ such that if $\operatorname{supp} \mu \subset[0, a]$ and $\operatorname{supp} \nu \subset[0, b]$, then

$$
\|\mu * \nu\| \leq B(a+b)\|\mu\|\|\nu\| .
$$

(ii) If $\gamma=0, \alpha \geq-1 / 2$, and $\kappa \in\{e, o\}$ then $*$ is a commutative, associative product on $M$ which is both weakly and norm continuous; i.e., there is a constant $B$ such that

$$
\|\mu * \nu\| \leq B\|\mu\|\|\nu\| \quad(\mu, \nu \in M) .
$$

(iii) If $\gamma=0,-1 / 2 \leq \alpha \leq 1 / 2$, and $\kappa \in\{e, o\}$ then $*$ is a positive commutative and associative convolution, i.e.,

$$
\mu, \nu \in M_{+} \Longrightarrow \mu * \nu \in M_{+} .
$$

(iv) If $\gamma=0,-1 / 2 \leq \alpha \leq 1 / 2$, and $\kappa=e$, then $(M, *)$ is a commutative Banach algebra with identity $\delta_{0}$ and with $\left(M_{1}, *\right)$ as a semigroup. Indeed, $(M, *)$ is a hermitian hypergroup (DJS hypergroup); for each $\lambda \in \Lambda, \phi_{\lambda}$ is a character in the sense that the mapping $\mu \mapsto \widehat{\mu}(\lambda)$ is a homomorphism.

(For information on hypergroups, see [CMS93] and the references cited there.)

3.2. Renormalization. In this section we continue to suppress superscripts and subscripts and we assume

$$
\gamma=0, \quad-1 / 2 \leq \alpha \leq 1 / 2, \quad \kappa \in\{e, o\}
$$

and

$$
\lambda_{0}=0 \quad \text { or } \quad \lambda_{0}=\mu i \text { with } \mu^{2} \geq \alpha^{2}-1 / 4
$$

so that $\tau_{\lambda_{0}}$ is positive on $(0, \infty)$ by Lemma 3.1. Define

$$
\psi_{\lambda}(x)= \begin{cases}\mathcal{T}_{\lambda}(x) / \mathcal{T}_{\lambda_{0}}(x) & \text { if } 0<x<\infty \\ 1 & \text { if } x=0\end{cases}
$$

Then $\psi_{\lambda}$ is a continuous function on $[0, \infty)$ because continuity at $x=0$ in the case of $\kappa=o$ follows by L'Hôpital's rule. Thus it is possible to define measures by

$$
d \tau_{x, y}(\xi)=\frac{\mathcal{T}_{\lambda_{0}}(\xi)}{\mathcal{T}_{\lambda_{0}}(x) \mathcal{T}_{\lambda_{0}}(y)} d \sigma_{x, y}(\xi) \quad(0<x, y<\infty)
$$

and

$$
\tau_{x, 0}=\tau_{0, x}=\delta_{x} \quad(x \in[0, \infty))
$$

Thus

$$
\psi_{\lambda}(x) \psi_{\lambda}(y)=\int \psi_{\lambda} d \tau_{x, y} \quad(\lambda \in \mathbb{C})
$$


holds for all $x, y \in[0, \infty)$. Now $\psi_{\lambda_{0}}(x)=1$ for all $x$ so if $\lambda=\lambda_{0}$ in (42) it follows from 4 in Theorem 3.2 that $\tau_{x, y} \in M_{1}$. Define the operation $\star$ on $M$ by

$$
\int f d(\mu \star \nu)=\iint\left(\int f d \tau_{x, y}\right) d \mu(x) d \mu(y)
$$

for $f \in C_{B}$ and $\mu, \nu \in M$. Thus, we easily obtain the following

Theorem 3.4. Suppose $\gamma=0,-1 / 2 \leq \alpha \leq 1 / 2$, and $\kappa \in\{e, o\}$, and that $\lambda_{0}=0$ or $\lambda_{0}=i \mu$ with $\mu^{2} \geq \alpha^{2}-1 / 4$. Then $(M, \star)$ is a commutative Banach algebra with identity $\delta_{0}$ and with $\left(M_{1}, \star\right)$ as a semigroup. Indeed, $(M, \star)$ is a hermitian hypergroup (DJS hypergroup); every bounded real $\psi_{\lambda}$ is a character in the sense that the mapping $\mu \mapsto \int \psi_{\lambda} d \mu$ is a homomorphism.

Remark. When $\gamma>0, \phi_{\lambda}$ must have zeros (Lemma 3.1), so renormalization is impossible in those cases.

4. Particular cases. The choice of particular values of the parameters $\gamma, \alpha$, and $\kappa$ in Theorem 2.1 lead to a number of historically important special cases. The formulas for the Jacobi functions are included in Corollary 2.2. The cases $\alpha= \pm 1 / 2$ are included explicitly because they are historically of interest and because the formulas are strikingly simple. Other cases are included because they yield familiar formulas in an interesting way.

Recall the Bessel function of the first kind with real and imaginary arguments:

$$
J_{n}(x)=\sum_{k=0}^{\infty} \frac{(-1)^{k}(x / 2)^{2 k+n}}{k !(k+n) !} \quad I_{n}(x)=i^{-n} J_{n}(i x) .
$$

4.1. Even boundary conditions. For the even boundary conditions, the formula given in Theorem 2.1 leads to the following cases:

1.a). When $\gamma \geq 0$ and $\alpha=-1 / 2$ the oblate radial functions become the so-called "modified Mathieu functions" which satisfy (12) with

$$
\begin{aligned}
\mathcal{K}_{o b l}^{-1 / 2, \gamma}(\xi ; x, y) & =\frac{1}{4} \sinh \xi \sinh x \sinh y \sum_{k=1}^{\infty} \frac{\gamma^{k}}{k !(k-1) !}\left(\frac{\chi_{0}}{4}\right)^{k-1} \\
& =\sinh \xi \sinh x \sinh y \sqrt{\frac{\gamma}{4 \chi_{0}}} I_{1}\left(\sqrt{\gamma \chi_{0}}\right)
\end{aligned}
$$

1.b). If $\gamma \geq 0$ and $\alpha=1 / 2$ then

$$
\mathcal{K}_{o b l}^{1 / 2, \gamma}(\xi ; x, y)=\tanh \xi \tanh x \tanh y \sqrt{\frac{\gamma}{4 \chi_{0}}} I_{1}\left(\sqrt{\gamma \chi_{0}}\right) .
$$

1.c). If $\gamma=0$ and $\alpha=-1 / 2,(12)$ reduces to the addition formula for cosines

$$
\mathcal{T}_{e, \lambda}^{-1 / 2,0}(x)=\phi_{\lambda}^{(-1 / 2,-1 / 2)}(x)=\cos \lambda x
$$

so $(12)$ reduces to

$$
\cos \lambda x \cos \lambda y=\frac{1}{2}[\cos \lambda(x-y)+\cos \lambda(x+y)] .
$$

and Theorem 3.4 yields the hypergroup structure on $M$ in which

$$
\delta_{x} * \delta_{y}=\frac{1}{2} \delta_{|x-y|}+\frac{1}{2} \delta_{x+y}
$$

which is equivalent in a natural way to the algebra of even measures on $\mathbb{R}$. 
1.d). If $\gamma=0$, and $\alpha=\frac{1}{2}$, the functions are

$$
\mathcal{T}_{e, \lambda}^{1 / 2,0}(x)=\frac{\cos \lambda x}{\cosh x}
$$

(12) then becomes

$$
\frac{\cos \lambda x}{\cosh x} \cdot \frac{\cos \lambda y}{\cosh y}=\frac{\cosh |x-y|}{2 \cosh x \cosh y} \cdot \frac{\cos \lambda|x-y|}{\cosh |x-y|}+\frac{\cosh (x+y)}{2 \cosh x \cosh y} \cdot \frac{\cos \lambda(x+y)}{\cosh (x+y)}
$$

When $\lambda=(\alpha+1 / 2) i=i$, the above formula reduces to the addition formula for hyperbolic cosines:

$$
1=\frac{\cosh (x-y)}{2 \cosh x \cosh y}+\frac{\cosh (x+y)}{2 \cosh x \cosh y}
$$

and Theorem 3.4 leads to the cosh hypergroup.

4.2. Odd boundary conditions. For the odd boundary conditions, (13) leads to the following special cases.

2.a). If $\gamma \geq 0$ and $\alpha=-1 / 2$, then

$$
\mathcal{M}_{o b l}^{-1 / 2, \gamma}(\xi ; x, y)=\frac{1}{2} \sum_{k=0}^{\infty} \frac{1}{(k !)^{2}}\left(\frac{\gamma \chi_{0}}{4}\right)^{k}=\frac{1}{2} I_{0}\left(\sqrt{\gamma \chi_{0}}\right)
$$

2.b). If $\gamma \geq 0$ and $\alpha=1 / 2$, then

$$
\mathcal{M}_{o b l}^{1 / 2, \gamma}(\xi ; x, y)=\frac{1}{2 \cosh \xi \cosh x \cosh y} I_{0}\left(\sqrt{\gamma \chi_{0}}\right)
$$

2.c). When $\gamma=0$ and $\alpha=-1 / 2$, the odd functions simplify to

$$
\mathcal{T}_{o, \lambda}^{-1 / 2,0}(x)=\sinh x \phi_{\lambda}^{\left(\frac{1}{2},-\frac{1}{2}\right)}(x)=\sinh x \phi_{2 \lambda}^{\left(\frac{1}{2}, \frac{1}{2}\right)}(x / 2)=\frac{\sin \lambda x}{\lambda},
$$

and (13) implies

$$
\frac{\sin \lambda x}{\lambda} \cdot \frac{\sin \lambda y}{\lambda}=\int_{|x-y|}^{x+y} \frac{\sin \lambda \xi}{\lambda} \frac{d \xi}{2} .
$$

In this case, renormalization with $\lambda_{0}=0$ yields

$$
\psi_{\lambda}(x)=\frac{\sin \lambda x}{\lambda x}=\sqrt{\frac{\pi}{2}} \frac{J_{1 / 2}(\lambda x)}{\sqrt{\lambda x}}
$$

so this is a special case of the product formula for Bessel functions of the first kind and Theorem 3.4 yields a hypergroup sructure on $M$ which is equivalent in a natural way to the algebra of rotationally invariant measures on $\mathbb{R}^{3}$.

2.d). When $\gamma=0$ and $\alpha=1 / 2$, then

$$
\mathcal{T}_{o, \lambda}^{1 / 2,0}(x)=\sinh x \phi_{\lambda}^{\left(\frac{1}{2}, \frac{1}{2}\right)}(x)=\frac{\sin \lambda x}{\lambda \cosh x}
$$


and Theorem 2.1 implies

$$
\frac{\sin \lambda x}{\lambda \cosh x} \frac{\sin \lambda y}{\lambda \cosh y}=\int_{|x-y|}^{x+y} \frac{\sin \lambda \xi}{\lambda \cosh \xi} \cdot \frac{1}{2 \cosh \xi \cosh x \cosh y} \cosh ^{2} \xi d \xi .
$$

or

$$
\frac{\sin \lambda x}{\lambda}, \frac{\sin \lambda y}{\lambda}=\int_{|x-y|}^{x+y} \frac{\sin \lambda \xi}{\lambda} \frac{d \xi}{2} .
$$

\section{REFERENCES}

[CMS93] W. C. Connett, C. Markett, And A. L. Schwartz, Product formulas and convolutions for angular and radial spheroidal wave functions, Trans. Amer. Math. Soc., 338 (1993), pp. 695-710.

[EMot53] A. Erdelyi, W. Magnus, F. Oberhettinger, and F. G. Tricomi, Higher Transcendental Functions, Volume I, McGraw-Hill Book Company, New York, 1953.

[FK73] M. FLENSTED-JENSEN AND T. KOORNWINDER, The convolution structure for Jacobi function expansions, Ark. Mat., 11 (1973), pp. 245-262.

[Fle72] M. FlENSTED-JENSEN, Paley-Wiener type theorems for a differential operator connected with symmetric spaces, Ark. Mat., 10 (1972), pp. 143-162.

[Hen57] P. HENRICI, A survey of I. N. Vekua's theory of elliptic partial differential equations with analytic coefficients, Z. Angew. Math. Phys., 8 (1957), pp. 169-203.

[Koo84] T. H. KooRNWINDER, Jacobi functions and analysis on noncompact semisimple lie groups, in Special Functions: Group Theoretical Aspects and Applications, R. A. Askey, T. H. Koornwinder, and W. Schempp, ed., Dordrecht, Reidel, 1984, pp. $1-85$. 
